\title{
Left-Handed Interfaces for Electromagnetic Surface Waves
}

\author{
A. V. Kats, ${ }^{1,2}$ Sergey Savel'ev, ${ }^{1,3}$ V. A. Yampol'skii, ${ }^{1,2}$ and Franco Nori ${ }^{1,4}$ \\ ${ }^{1}$ Frontier Research System, The Institute of Physical and Chemical Research (RIKEN), Wako-shi, Saitama, 351-0198, Japan \\ ${ }^{2}$ A.Ya. Usikov Institute for Radiophysics and Electronics Ukrainian Academy of Sciences, 61085 Kharkov, Ukraine \\ ${ }^{3}$ Department of Physics, Loughborough University, Loughborough LE11 3TU, United Kingdom \\ ${ }^{4}$ MCTP, CSCS, Physics Department, University of Michigan, Ann Arbor, Michigan 48109, USA
}

(Received 13 September 2006; published 16 February 2007)

\begin{abstract}
We show that surface electromagnetic waves (SEMWs) propagating along two-dimensional (2D) interfaces separating different metamaterials can behave analogously to 3D electromagnetic waves in either usual or left-handed media, depending on the permeabilities and/or permittivities of the two materials forming the interface. We derive the conditions when SEMWs carry energy opposite to the phase velocity. In analogy to three-dimensional (3D) left-handed media, we derive both an anomalous Cherenkov emission and a reversed Doppler effect. We also predict a negative refraction at the boundary between two different interfaces, which can be useful for perfect 2D lensing.
\end{abstract}

DOI: 10.1103/PhysRevLett.98.073901

PACS numbers: $42.25 .-\mathrm{p}$

Studies of surface waves in condensed matter began at the end of the 19th century, since the works of Lord Rayleigh, Zenneck, and Sommerfeld. In contrast to bulk waves, surface waves are localized near interfaces separating two media and their magnitudes decay quickly away from the interface. Surface waves manifest themselves in a wide variety of phenomena, including tsunami, earthquakes, as well as resonance anomalies in both light reflection [1] and transmission [2]. Electromagnetic surface waves are of general interest and can be observed in different materials [1,3], including semiconductors, metals, magnetic materials, and superconductors. Electromagnetic waves (EMWs) can be excited, e.g., by moving or oscillating charges close to the interface, or from bulk electromagnetic waves scattering at periodic structures. In particular, two specific methods involving attenuated total internal reflection are widely used [1].

Currently, there is a surge of interest in both surface [4] and bulk [5] waves propagating through artificial metamaterials. For surface waves, their resonant excitations (Wood-type anomalies) in nanofabricated hole arrays and other periodic and nonperiodic structures allow, e.g., to control the transparency of opaque films [2], to develop 2D waveguides for optoelectronics, and to amplify electromagnetic fields near sample boundaries [6]. For bulk waves, enormous progress has been achieved in fabricating left-handed materials (LHMs): transparent media with negative both permeability and permittivity $(\varepsilon<0, \mu<$ 0 ) (e.g., Refs. [5,7]). In contrast to ordinary transparent right-handed ( $\varepsilon>0, \mu>0$ ) materials (RHMs), the energy transport through bulk LHMs is opposite to the phase velocity, the Doppler shift is reversed, and the Cherenkov cone is inverted [5]. This promises several important applications, including perfect lensing (subwavelength image reconstruction) and novel waveguiding [5].

Comparing surface and bulk waves, a general question arises: can surface electromagnetic waves exhibit behavior analogous to bulk electromagnetic waves in LHM? Here, we derive conditions for the total energy flux of surface electromagnetic waves (SEMWs) to be antiparallel to its wave vector, as it is for bulk waves in LHM. For these surface waves, we also predict an inverse Doppler shift, an inverted Cherenkov angle, negative refraction, and negative group velocity with respect to the phase velocity. Thus, we propose new left-handed surface waves, which could be useful for perfect 2D lensing and novel wave guiding.

Surface waves. - Consider an interface $z=0$ separating two media, + and - , shown in Fig. 1. Subindexes + and refer to $z>0$ and $z<0$, respectively. The electromagnetic field of surface localized modes with in-plane wave vector $\mathbf{q}=\left(q_{x}, q_{y}\right)$, frequency $\omega$, and decay constants $p_{ \pm}>0$ has the form $\mathbf{E}_{ \pm}=\mathbf{E}_{ \pm}^{(0)} \exp \left(i \mathbf{q} \cdot \mathbf{r}_{\perp} \mp p_{ \pm} z-i \omega t\right)$ and $\mathbf{H}_{ \pm}=\mathbf{H}_{ \pm}^{(0)} \exp \left(i \mathbf{q} \cdot \mathbf{r}_{\perp} \mp p_{ \pm} z-i \omega t\right), \quad$ where $p_{ \pm}=$ $\sqrt{\mathbf{q}^{2}-\varepsilon_{ \pm} \mu_{ \pm} \omega^{2} / c^{2}}, \operatorname{Re}\left(p_{ \pm}\right)>0$, and $\mathbf{r}_{\perp}=(x, y)$. There exists a transverse electric (TE) [magnetic (TM)] polarized eigenmode with in-plane electric field $\mathbf{E}$ [magnetic field $\mathbf{H}]$ orthogonal to the wave vector $\mathbf{q}$, as shown in Fig. 1. The polarization of a TE mode is defined by $\mathbf{E}_{ \pm}^{(0)}=\mathbf{e} E^{(0)}$, $\mathbf{H}_{ \pm}^{(0)}=\left(c / \omega \mu_{ \pm}\right)\left[\mathbf{k}_{ \pm} \times \mathbf{e}\right] E^{(0)}, \mathbf{k}_{ \pm}=\left(\mathbf{q}, \pm i p_{ \pm}\right)$, with the unit polarization vector $\mathbf{e}=\mathbf{q} \times \mathbf{e}_{z} / q$. Because of the duality of Maxwell equations, TM-type SEMWs can be derived from TE type by exchanging: $\varepsilon \leftrightarrow \mu, \mathbf{H} \rightarrow-\mathbf{E}$, $\mathbf{E} \rightarrow \mathbf{H}$. We present the equations for these TE waves.

Matching the tangential components of the electric and magnetic fields at the interface implies that $p_{+} / \mu_{+}+$ $p_{-} / \mu_{-}=0$ for the TE SEMW, which can be rewritten as a dispersion relation

$$
\mathbf{q}^{2}(\omega)=\left(\frac{\omega^{2}}{c^{2}}\right) \frac{\varepsilon_{+} / \mu_{+}-\varepsilon_{-} / \mu_{-}}{1 / \mu_{+}^{2}-1 / \mu_{-}^{2}} .
$$

We can neglect the spatial dispersion, but assume the permeabilities $\mu_{ \pm}(\omega)$ and permittivities $\varepsilon_{ \pm}(\omega)$ to be frequency dependent, which is important for both usual plasmon polaritons [1] and surface waves in artificial media 

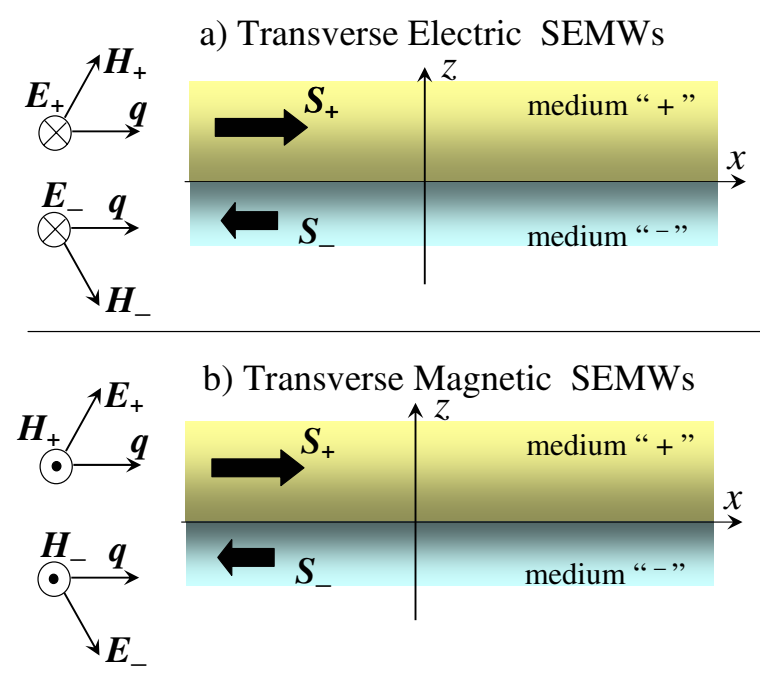

FIG. 1 (color online). Schematic diagram of the relative orientations of the electric $\mathbf{E}_{ \pm}^{(0)}$ and magnetic $\mathbf{H}_{ \pm}^{(0)}$ field amplitudes respective to the wave vector $\mathbf{q}$ and energy flux densities $\mathbf{S}_{ \pm}$in the neighboring media for TE (a) and TM (b) surface waves. The color gradient illustrates the decay of the SEMWs away from the interface. Note that for interfaces separating any kind of media (for instance, usual dielectric and metal, but not only LHM and RHM), the vectors $\operatorname{Re} \mathbf{E}\left(\mathbf{r}_{\perp}, z, t\right), \operatorname{Re} \mathbf{H}\left(\mathbf{r}_{\perp}, z, t\right)$, and $\mathbf{q}$ form the right triplet in one and the left triplet in the other media. Moreover, the Pointing vector is parallel to $\mathbf{q}$ in one media and antiparallel in the other.

[8]. This frequency dependence and $q$ independence of $\varepsilon$ 's and $\mu$ 's are valid from $\mathrm{GHz}$ to optical frequencies. Requiring $p_{ \pm}^{2}>0, \mathbf{q}^{2}>0$, and $p_{ \pm}>0$ results in the necessary conditions for the existence of TE SEMWs: $\mu_{-} \mu_{+}<0 ; \quad\left(\mu_{+} \varepsilon_{+}-\varepsilon_{-} \mu_{-}\right) /\left(\mu_{-}^{2}-\mu_{+}^{2}\right)>0 ; \quad$ and $\left(\varepsilon_{-} \mu_{+}-\varepsilon_{+} \mu_{-}\right) /\left(\mu_{-}^{2}-\mu_{+}^{2}\right)>0$.

Note that if we consider small active losses, then the dielectric permittivity $\varepsilon=\varepsilon^{\prime}+i \varepsilon^{\prime \prime}$ can have an imaginary part, $\varepsilon^{\prime \prime} \ll\left|\varepsilon^{\prime}\right|$; therefore, a small imaginary part $q^{\prime \prime}$ of the wave number $q$ appears in Eq. (1), i.e., $q=q^{\prime}+i q^{\prime \prime}$, $q^{\prime \prime} \ll q^{\prime}$ with $q^{\prime \prime} \propto \varepsilon^{\prime \prime}$. Thus, small losses result in a finite SEMW's mean free-path length $L \propto 1 / q^{\prime \prime} \propto 1 / \varepsilon^{\prime \prime}, q^{\prime} L \gg$ 1. For experiments, it is necessary to deal with distances which do not significantly exceed $L$. For a standard metal at room temperature, one could use $\varepsilon^{\prime \prime} / \varepsilon^{\prime} \sim \nu / \omega_{p}$, where the electron collision frequency $\nu$ is about $10^{12}-10^{14} \mathrm{~s}^{-1}$ and the plasma frequency $\omega_{p}$ is about $10^{16} \mathrm{~s}^{-1}$. This results in $L \sim\left(\omega_{p} / \omega\right)^{3} c / \nu \approx 0.1-1 \mathrm{~mm}$, depending on the material, temperature, and frequency (which is taken in the optical range). For simplicity, below we consider lossless materials.

Direction of energy propagation. - The energy flux density (determined by the time-averaged Pointing vector $\left.\mathbf{S}=c \operatorname{Re}\left[\mathbf{E}^{(0)} \times \mathbf{H}^{(0) *}\right] / 8 \pi\right)$ is directed in opposite directions in neighboring media, and both decay exponentially away from the interface: $\mathbf{S}_{ \pm}=\left(c^{2} \mathbf{q} / 8 \pi \omega \mu_{ \pm}\right)\left|E^{(0)}\right|^{2} \times$ $\exp \left(-2 p_{ \pm}|z|\right)$, where $E$ is the complex amplitude of the electric field of the wave. Moreover, for SEMWs as shown in Fig. 1, the real electric field $\operatorname{Re} \mathbf{E}(\mathbf{r}, t)$, real magnetic field $\operatorname{Re} \mathbf{H}(\mathbf{r}, t)$, and the wave vector $\mathbf{q}$ (all vectors have only real components) form a left-handed triplet in one medium (say, medium "-") and a right-handed triplet in the other medium "+" at any point in space and time, since there is no phase shift between $\mathbf{E}(\mathbf{r}, t)$ and $\mathbf{H}(\mathbf{r}, t)$. Therefore, the Veselago's definition [7] for 3D LHM, using triplets of vectors, cannot be directly applied for $2 \mathrm{D}$ interfaces. However, all the unusual properties of electromagnetic waves in LHM are related to energy transport directed opposite to the phase velocity. Therefore, the natural generalization of the concept of LHM to the 2D case can be done in terms of the backward total energy flux relative to the phase velocity.

To derive the total energy flux $\overline{\mathbf{S}}$ carried by the TE SEMW, we integrate over $z$ the Pointing vector $\mathbf{S}$ :

$$
\overline{\mathbf{S}}=\frac{c^{2} \mathbf{q}}{16 \pi \omega} \frac{1}{\mu_{+} p_{+}}\left[1-\frac{\mu_{+}^{2}}{\mu_{-}^{2}}\right]\left|E^{(0)}\right|^{2}
$$

assuming $\mu_{+}>0$. Consequently, $\overline{\mathbf{S}}$ is parallel (antiparal-

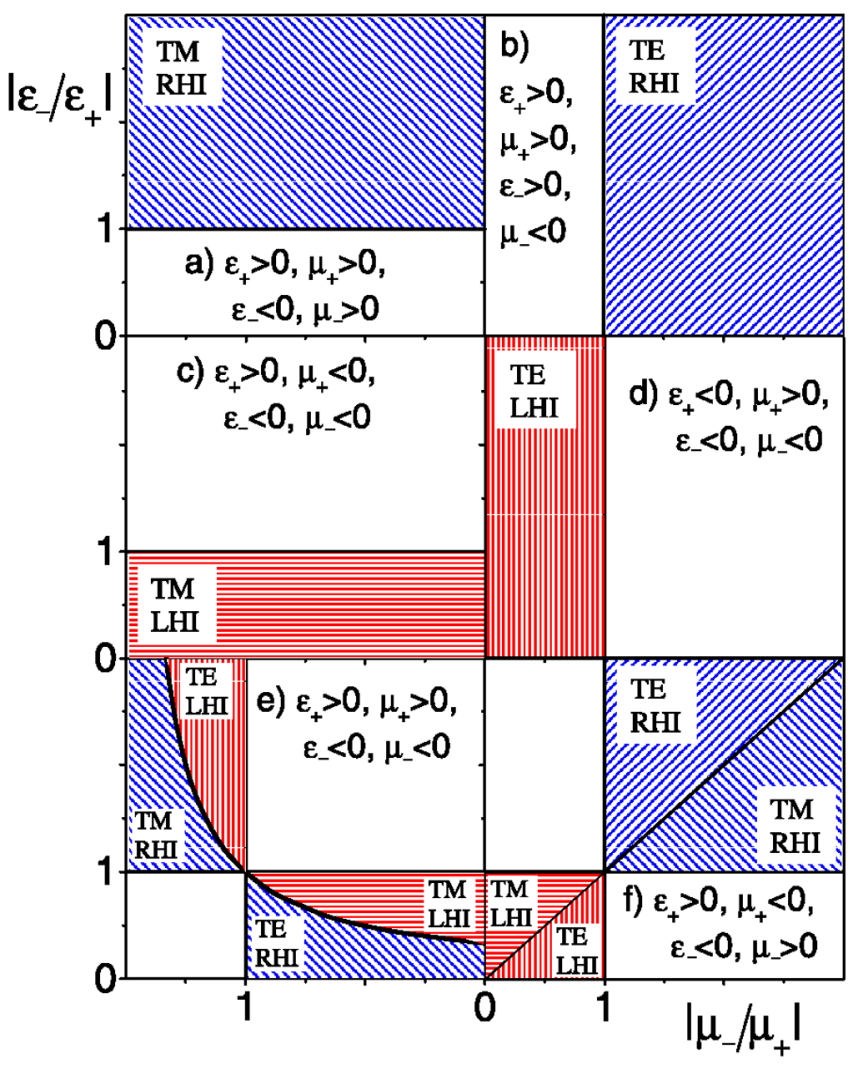

FIG. 2 (color online). Regions in the $\left(\left|\varepsilon_{-} / \varepsilon_{+}\right|,\left|\mu_{-} / \mu_{+}\right|\right)$ plane where TE and TM SEMWs exist are marked using different patterns. No surface waves occur in the white regions. Red (blue) regions correspond to left-handed (right-handed) interfaces LHI (RHI): (a) RHM-to-metal, (b) RHM-to-meta-dielectric, (c) meta-dielectric-to-LHM, (d) metal-to-LHM, (e) RHM-toLHM, (f) metal-to-meta-dielectric. For cases (c)-(f), there exist regions where LHI can be realized. The hyperbola in panel (e) is $\left|\varepsilon_{+} / \varepsilon_{-}\right|=\left|\mu_{-} / \mu_{+}\right|$. 
lel) to the wave vector $\mathbf{q}$ for $\mu_{+}^{2}<\mu_{-}^{2}\left(\mu_{+}^{2}>\mu_{-}^{2}\right)$. Therefore, the TE SEMW behaves as a wave in a LHM when $\mu_{+}^{2}>\mu_{-}^{2}$, where $\mu_{+}>0$ and $\mu_{-}<0$. It is natural to call such interfaces as left-handed interfaces (LHIs). Similarly, for TM SEMW, LHIs occur for $\varepsilon_{+}^{2}>\varepsilon_{-}^{2}$, if $\varepsilon_{+}>0, \varepsilon_{-}<0$. Figure 2 summarizes the conditions when SEMWs exist and their total energy flux is either parallel (for right-handed interfaces, RHIs) or antiparallel (for LHIs) to the phase velocity for all possible (6 types) interfaces depicted in six panels, (a)-(f).

Anomalous Doppler shift and Cherenkov radiation.Here, we consider a source of radiation (that generates SEMWs) moving with velocity $\mathbf{v}$ along an interface. If the receiver is behind the source, it only measures the energy flux emitted backwards, i.e., with $\overline{\mathbf{S}} \cdot \mathbf{v}<0$. For left-handed interfaces, the wave vector $\mathbf{q}$ is opposite to $\overline{\mathbf{S}}$, resulting in $\mathbf{q} \cdot \mathbf{v}>0$. This means that the Doppler shift of the frequency $\omega$, determined by $\mathbf{q} \cdot \mathbf{v}$, is reversed with respect to the corresponding Doppler shift of the SEMWs propagating on right-handed interfaces, where $\mathbf{q} \cdot \mathbf{v}<0$ follows from $\overline{\mathbf{S}} \cdot \mathbf{v}<0$ and the fact that $\overline{\mathbf{S}}$ and $\mathbf{q}$ point in the same direction. In other words, in a LHI, the measured frequency increases (decreases) relative to the emitted frequency, if the source and receiver move away from (approach to) each other.

For the 2D case, the Cherenkov cone of radiation, produced by a charge moving with velocity $\mathbf{v}$ along the interface, is replaced by a Cherenkov angle. In analogy to the 3D case, the Cherenkov condition $\omega-\mathbf{q} \cdot \mathbf{v}=0$ for SEMWs defines an angle $\theta_{\text {phase }}$ between the phase velocity $v_{\text {phase }}$ of the wave and the charge velocity $\mathbf{v}, \cos \theta_{\text {phase }} \equiv$ $\mathbf{q} \cdot \mathbf{v} /(q v)=\omega /(q v)$. For left-handed media, $\overline{\mathbf{S}}$ and $\mathbf{q}$ are antiparallel; thus, the angle $\theta_{\text {energy }}$ between the direction of the energy flux and $\mathbf{v}$ [i.e., $\cos \left(\theta_{\text {energy }}\right)=\overline{\mathbf{S}} \mathbf{v} /(\bar{S} v)$ ] is $\theta_{\text {energy }}=\theta_{\text {phase }}+\pi$, i.e., the Cherenkov angle is inverted.

Negative refraction of SEMWs. - In analogy to the negative refraction of 3D waves on the boundary between RHM and LHM, SEMWs should refract negatively on the boundary between RHIs and LHIs. In order to see this, consider two interfaces at $z=0$ separated by the boundary $x=0$, Fig. 3. The energy flux of the incident SEMW, say, from the RHI $(x<0)$ continues to propagate in the LHI $(x>0)$ from the left to the right; i.e., the $x$ components of the energy fluxes of both incident and transmitted (refracted) waves are positive. Since the wave vector $\mathbf{q}$ is parallel to the energy flux in the RHI and antiparallel in the LHI, the component $q_{x}$ perpendicular to the boundary $x=0$ has, in contrast, different signs for $x>0$ and $x<0$. This evidently should result in negative refraction of SEMWs at the boundary between these two interfaces.

As an example, we consider the boundary between two interfaces, each separating two lossless opaque media (i.e., $\left.\varepsilon_{ \pm} \mu_{ \pm}<0\right)$. Interface 1 separates metal-like media [occupying the top-left corner, $x<0, z>0$ in Fig. 3(a) and 3(c)] with $\varepsilon_{1+}<0$ and $\mu_{1+}>0$ from an artificial nontransparent dielectric [situated in the bottom-left region, $x<0, z<$

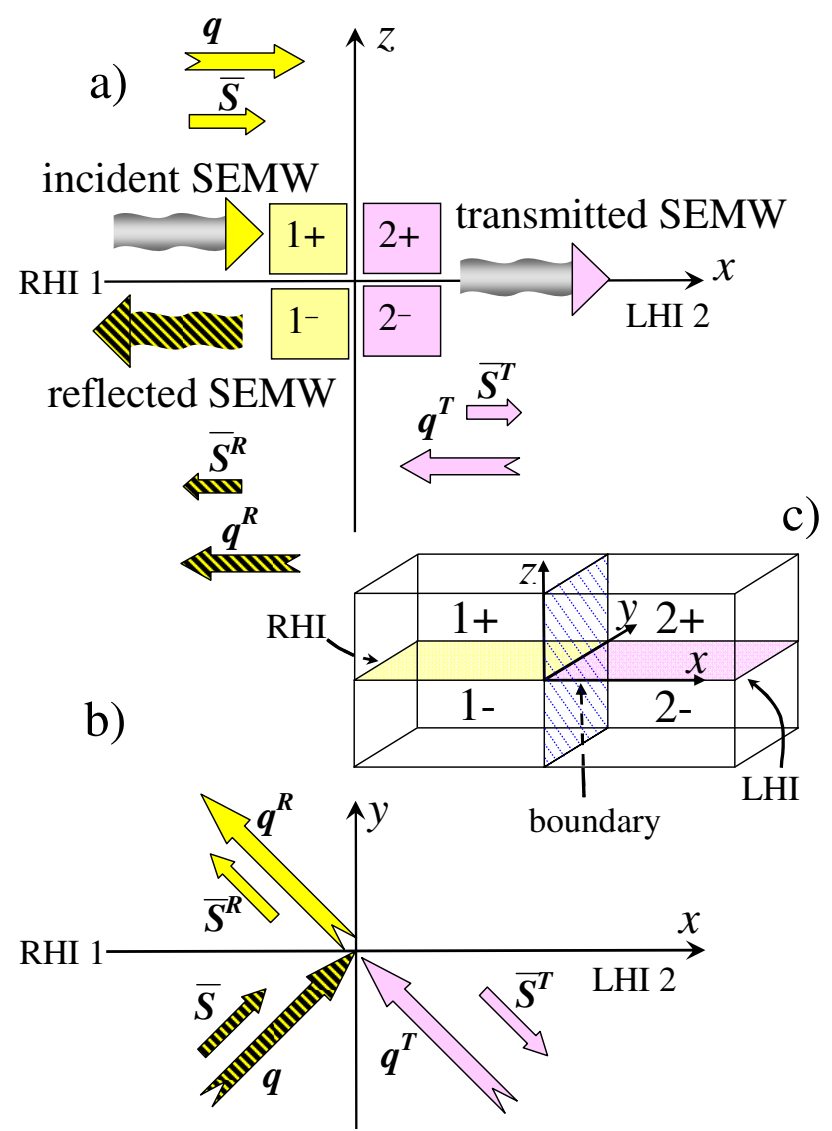

FIG. 3 (color online). Reflection and refraction of a SEMW at the boundary $x=0$ between a RHI at $x<0$ and a LHI at $x>0$. The wave vectors and energy fluxes are schematically shown as a side view (a), top view (b), and 3D diagram (c).

0 in Fig. 3(a) and 3(c)] with $\varepsilon_{1-}>0$ and $\mu_{1-}<0$. Interface 2, occupying the right half-space $x>0$, is formed by media where the artificial dielectric $\left(\varepsilon_{2+}>0 ; \mu_{2+}<0\right)$ is located on top of the metal $\left(\varepsilon_{2-}<0 ; \mu_{2-}>0\right)$. These interfaces [8] support both TM and TE surface waves in different frequency bands.

Let us now consider a monochromatic TE-type SEMW incident on the boundary $x=0$ from the left interface as well as outgoing reflected and refracted waves. The electromagnetic fields $\mathbf{E}_{ \pm}^{i}(x<0), \mathbf{E}_{ \pm}^{R}(x<0)$, and $\mathbf{E}_{ \pm}^{T}(x>0)$ of these waves have the form

$$
\mathbf{E}_{ \pm}=C \mathbf{e} \exp \left[i\left(\mathbf{q} \cdot \mathbf{r}_{\perp}-\omega t\right)\right] \exp \left(\mp p_{ \pm} z\right)
$$

with $p_{ \pm}=p_{1 \pm}$ for $x<0$, or $p_{ \pm}=p_{2 \pm}$ for $x>0$. The amplitude coefficient $C$ equals $1, R$, or $T$; the unit polarization vector $\mathbf{e}$ can be $\mathbf{e}^{i}, \mathbf{e}^{R}$, or $\mathbf{e}^{T}$; and the wave vector is $\mathbf{q}, \mathbf{q}^{R}$, or $\mathbf{q}^{T}$, for incident, reflected, and transmitted waves, respectively. Because of the homogeneity in the $y$ direction, the $y$ components of the wave vectors $\mathbf{q}^{R}$ and $\mathbf{q}^{T}$ of the reflected and transmitted waves coincide with that of the incident wave, $q_{y}^{R}=q_{y}^{T}=q_{y}$, while the moduli of $\mathbf{q}^{R}$ and $\mathbf{q}^{T}$ are defined by the dispersion relations of the corresponding interfaces. These conditions determine the $x$ 


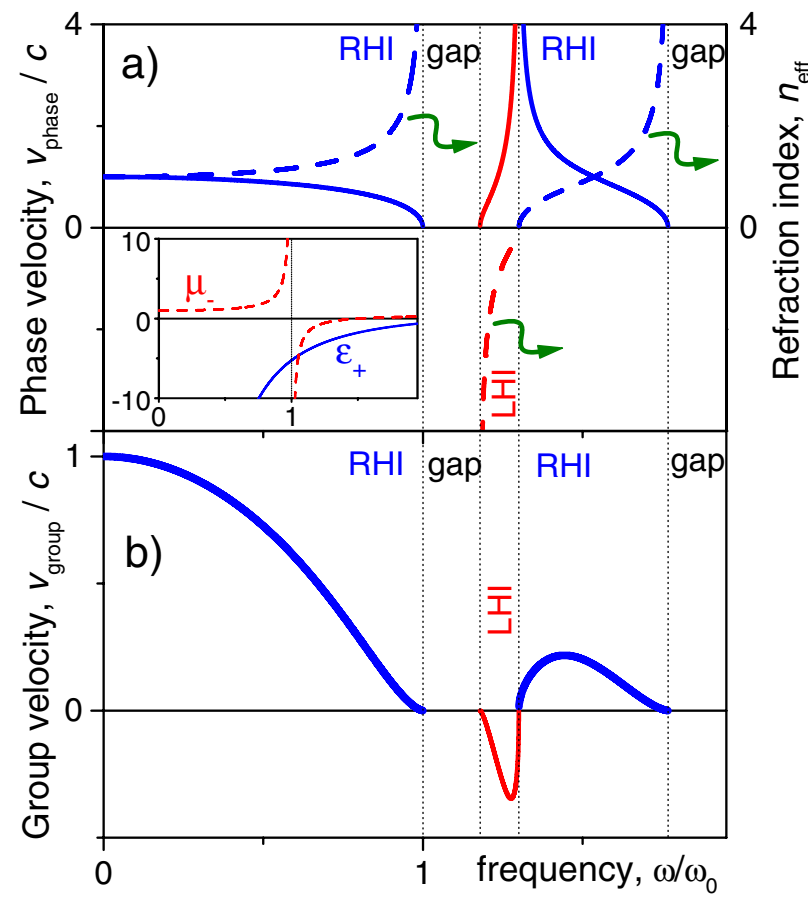

FIG. 4 (color online). Frequency dependence of the refraction index $n_{\text {eff }}$ [dashed line in (a)], phase velocity $v_{\text {phase }}$ [solid line in (a)], and group velocity $v_{\text {group }}$ [in (b)] for surface waves propagating along an interface separating media with permittivities $\varepsilon_{+}, \varepsilon_{-}$and permeabilities $\mu_{+}, \mu_{-}$. Also, $\varepsilon_{+}$and $\mu_{-}$are shown in the inset; and $\varepsilon_{-}=\mu_{+}=1$. Both TE and TM modes exist in different frequency regions. The frequency interval where the group velocity is negative (multiplied by five, to see this better) corresponds to the LHI where the total energy flux is directed opposite to the phase velocity. The parameters used are $F=$ 0.56 , and $\omega_{p} / \omega_{0}=2.5$ (cf. [10] ).

component $q_{x}^{T}= \pm\left[\left(\mathbf{q}^{T}\right)^{2}-\left(q_{y}^{T}\right)^{2}\right]^{1 / 2}$ of the wave vector for the transmitted SEMW propagating along interface 2 . The sign of $q_{x}^{T}$ is defined by the continuity of the total energy flux and has to be negative if the boundary $x=0$ separates the RHI and LHI. Indeed, this corresponds to the energy fluxes of both reflected and transmitted waves being directed away from the boundary: $\bar{S}_{x}^{R}<0$ and $\bar{S}_{x}^{T}>0$.

For arbitrary permeabilities and permittivities, besides outgoing waves $\mathbf{E}_{ \pm}^{R}$ and $\mathbf{E}_{ \pm}^{T}$ from the boundary $x=0$, there exist additional electromagnetic fields localized in the vicinity of $x=0$. Thus, calculating the amplitudes of the reflected $R$ and transmitted $T$ waves is a rather complicated diffraction problem. Here we consider a particular case where interfaces satisfy specific conditions

$$
\mu_{2+} / \mu_{1+}=\mu_{2-} / \mu_{1-}=\varepsilon_{1-} / \varepsilon_{2-}=\varepsilon_{1+} / \varepsilon_{2+} .
$$

For these conditions (4), there are no localized modes; i.e., the electric and magnetic fields of the propagating waves, Eq. (3), obey the usual continuity conditions at the boundary $x=0$. When Eqs. (4) hold, the amplitudes of the reflected and transmitted waves can be easily derived, $R=$ $\left(\mu_{1+}+\mu_{2+}\right) /\left(\mu_{1+}-\mu_{2+}\right), \quad T=2 \mu_{2+} /\left(\mu_{1+}-\mu_{2+}\right)$. The corresponding energy coefficients, $\rho \equiv-\bar{S}_{x}^{R} / \bar{S}_{x}^{i}>0$ and $\tau \equiv \bar{S}_{x}^{T} / \bar{S}_{x}^{i}>0, \quad$ are $\rho=\left(\mu_{1+}+\mu_{2+}\right)^{2} /\left(\mu_{1+}-\right.$ $\left.\mu_{2+}\right)^{2}, \quad \tau=4\left|\mu_{2+} \mu_{1+}\right| /\left(\mu_{1+}-\mu_{2+}\right)^{2}$. Our prediction above provides a relatively simple way to achieve $2 \mathrm{D}$ perfect lensing of SEMWs. A more cumbersome structure, without LHIs, for 2D perfect lensing of SEMWs, was suggested in [9].

Dispersion relation and group velocity. - Consider here an example of a recently proposed [5] and experimentally realized (e.g., $[10,11])$ artificial medium with permeability $\mu_{-}(\omega)=1-F \omega^{2} /\left(\omega^{2}-\omega_{0}^{2}\right)$ neighboring with a metallike medium with permittivity $\varepsilon_{+}(\omega)=1-\omega_{p}^{2} / \omega^{2}$. Both $\varepsilon_{-}$and $\mu_{+}$are here assumed to be frequency-independent positive quantities. The permeability $\mu_{-}$is negative in the region $\omega_{0}<\omega<\omega_{0} / \sqrt{1-F}$ and, thus, we obtain a LHI for TE SEMWs. Namely, choosing, for instance, $\varepsilon_{-}=$ $\mu_{+}=1$ we obtain the effective refraction index $n_{\text {eff }}=$ $[c q(\omega) / \omega] \operatorname{sgn}(\mathbf{q} \cdot \overline{\mathbf{S}})$, phase velocity $v_{\text {phase }}=\omega / q(\omega)$, and group velocity $v_{\text {group }}=d \omega / d q$, shown in Fig. 4. The phase velocity exceeds the vacuum light speed $c$ in the frequency range where $-1<n_{\text {eff }}<0$. Remarkably, the calculated group velocity (Fig. 4) is negative (positive) for frequencies when the interface is left handed (right handed). This common situation, not stressed in the past, occurs now because the group velocity is directed along the total energy flux.

In conclusion, we show that $2 \mathrm{D}$ interfaces separating $3 \mathrm{D}$ metamaterials can exhibit properties of 2D left-handed media for surface waves. These waves are characterized by a total energy flux and group velocity antiparallel to the phase velocity and, therefore, should have a negative Doppler frequency shift, inverted Cherenkov angle, and negative refraction index.

We acknowledge partial support from the NSA, LPS, ARO, NSF Grant No. EIA-0130383, JSPS-RFBR No. 0602-91200, MEXT Grant-in-Aid No. 18740224, and the EPSRC via No. EP/D072581/1.

[1] H. Raether, Surface Plasmons (Springer, New York, 1988); Surface Polaritons, edited by V. M. Agranovich and D. L. Mills (North-Holland, Amsterdam, 1982).

[2] T. W. Ebbesen et al., Nature (London) 391, 667 (1998); A. V. Kats et al., Phys. Rev. B 72, 193405 (2005).

[3] S. Savel'ev et al., Phys. Rev. Lett. 95, 187002 (2005).

[4] W. L. Barnes et al., Nature (London) 424, 824 (2003).

[5] J.B. Pendry and D. R. Smith, Sci. Am. 295, No. 7, 60 (2006); J. B. Pendry, Contemp. Phys. 45, 191 (2004).

[6] K. Li et al., Phys. Rev. Lett. 91, 227402 (2003).

[7] V. G. Veselago, Sov. Phys. Usp. 10, 509 (1968).

[8] A. Alu et al., Phys. Rev. E 74, 016604 (2006).

[9] H. Shin and S. Fan, Phys. Rev. Lett. 96, 073907 (2006).

[10] R. A. Shelby et al., Science 292, 77 (2001).

[11] A. N. Grigorenko et al., Nature (London) 438, 335 (2005). 